\title{
Escovopsis kreiselii specialization to its native hosts in the fungiculture of the lower attine ant Mycetophylax morschi
}

\author{
Bruna Cristina Custodio • Andre Rodrigues
}

Received: 28 March 2018/Accepted: 29 August 2018/Published online: 11 September 2018

(C) Springer Nature Switzerland AG 2018

\begin{abstract}
Parasite-host associations are widespread in nature and the fungus-growing ants are considered model organisms to study such interactions. These insects cultivate basidiomycetous fungi for food, which are threatened by mycotrophic fungi in the genus Escovopsis. Although recently described from colonies of the lower attine ant Mycetophylax morschi, the biology and pathogenicity of Escovopsis kreiselii are unknown. Herein, we evaluated the interaction of E. kreiselii with fungi cultivated by M. morschi (native hosts) and with a fungus cultivated by another attine ant species (non-native host). In addition, we examined the physical interactions between hypha of $E$. kreiselii and hypha from its native hosts using scanning electron microscopy. Escovopsis kreiselii inhibited the growth of fungal cultivars by $24 \%$ or more (with exception of one isolate), when compared
\end{abstract}

Electronic supplementary material The online version of this article (https://doi.org/10.1007/s10482-018-1158-x) contains supplementary material, which is available to authorized users.

B. C. Custodio · A. Rodrigues

Department of Biochemistry and Microbiology, UNESP São Paulo State University, Av. 24-A, 1515, Rio Claro, SP 13506-900, Brazil

A. Rodrigues $(\bowtie)$

Center for the Study of Social Insects, UNESP - São

Paulo State University, Av. 24-A, 1515, Rio Claro,

SP 13506-900, Brazil

e-mail: andrer@rc.unesp.br to the fungal cultivars growing alone. Escovopsis kreiselii is attracted towards its native hosts through chemotaxis and inhibition occurs when there is physical contact with the hyphae of the fungal cultivar. As reported for Escovopsis parasites associated with leafcutter ants (higher attines), E. kreiselii growth increased in the presence of its native hosts, even before contact between both fungi occurred. In interactions with the fungal cultivar that is not naturally infected by E. kreiselii (non-native host), it caused inhibition but not at the same magnitude as in native hosts. Multiple lines of evidence suggest that $E$. kreiselii is an antagonist of the fungus cultivated by $M$. morschi and can chemically recognize such fungus.

Keywords Antagonism - Chemotaxis · Fungal interactions $\cdot$ Host-parasite

\section{Introduction}

Symbiotic interactions may be neutral, beneficial, or harmful to the individuals involved. In parasite-host systems, interactions are usually prolonged and can involve co-evolution between participants. A parasite lives in close association with its host, obtaining all or part of its nutrients from the host, exhibiting some degree of adaptive structural modifications and causing damage to the host (Price 1980). Understanding 
how these interactions are established and evolve is challenging, but can be studied using model systems. There are many examples of parasite-host systems in nature, including human parasitic protozoa transmitted by insects (Graczyk et al. 2005), larvae of freshwater mussels that parasitize fish (Horký et al. 2014), parasitic plants that infect other dicotyledonous plants (Kaiser et al. 2015), fungi that manipulate ant behaviour (Hughes et al. 2011), among others. One example of a complex parasite-host interaction occurs in colonies of fungus-farming ants that cultivate fungi for food.

Ants that practice fungiculture for food belong to the subtribe Attina (Hymenoptera: Formicidae: Attini, hereafter named "the attines"). These insects depend on mutualism with their fungal partners, where ants provide substrate for fungus cultivation and ensure fungal protection and dispersal. The fungus provides nutrients for ant colony survival (Weber 1972). While the higher attine ants can cultivate three distinct phylogenetic clades of fungi including Leucoagaricus gongylophorus (Agaricaceae, Mueller et al. 2018), the lower attine ants cultivate several lineages of Leucocoprinus fungi (Agaricaceae, Mueller et al. 1998), as well as fungi in the Pterulaceae family (Munkacsi et al. 2004).

The lower attines perform the oldest type of fungiculture (Schultz and Brady 2008), cultivating basidiomycetous fungi that can live outside association with ants (free-living). Such fungi are thought to be less specialized than the fungal cultivars of the higher attine ants, since they do not present adaptations such as gongylidia, vesicular structures present at the tip of the hyphae, which constitutes the primary food supply for the colony (Weber 1972; Mueller et al. 1998). Occasionally, lower attines domesticate new fungi from free-living stocks, thus the co-evolution between these ants and their cultivars is weak or absent (Kellner et al. 2017). On the other hand, within the higher attines, leafcutter ants are considered the most derived group of fungus-growing ants and co-evolved with at least two clades of basidiomycetous fungi (Mueller et al. 2018). It is thought that such fungi do not have a free-living existence and have adaptations to mutualism with ants (Chapela et al. 1994; Schultz and Brady 2008; Mueller et al. 2018). All attine ants cultivate their fungal partners in a structure known as the fungus garden. The garden matrix harbors a microbiome full of yeasts (Carreiro et al. 1997), bacteria (Kellner et al. 2015; Meirelles et al. 2016), and filamentous fungi (Reis et al. 2015; Pereira et al. 2016), including Escovopsis, a fungus genus considered as mycoparasites of the ant fungal cultivar (Currie et al. 1999).

Escovopsis presents wide morphological (different spore colors and other markers) and phylogenetic diversity, which is not entirely represented by the seven currently described species (Meirelles et al. 2015a; Birnbaum and Gerardo 2016). Morphologically distinct Escovopsis species infect different fungal cultivars, suggesting patterns of specialization are shaped by co-evolutionary processes in this parasite-host interaction (Gerardo et al. 2006; Birnbaum and Gerardo 2016). Escovopsis kreiselii (a pinkspored species) was isolated and described from colonies of the lower attine Mycetophylax morschi (Meirelles et al. 2015b). Although previous studies have evaluated the ecology of other pink-spored Escovopsis infecting fungal cultivars that are also infected by E. kreiselii (e.g. clades 1 and 2, G3 fungi according to Gerardo et al. 2006; Birnbaum and Gerardo 2016; Kellner et al. 2017), none of these isolates belong to the same monophyletic clade as $E$. kreiselii (Meirelles et al. 2015b). Furthermore, the physical interaction between E. kreiselii and the fungus cultivated by lower attine ants is unknown.

Herein, we explore the interactions of E. kreiselii with three fungal cultivars: two cultivated by $M$. morschi (its native hosts) and one cultivated by leafcutter ants, a non-native host. Combining in vitro bioassays with scanning electronic microscopy, we evaluated whether E. kreiselii inhibits its hosts, like other Escovopsis species. In addition, we assessed $E$. kreiselii specificity towards its native hosts. Finally, we evaluated the possible mechanisms that allow this antagonism to occur.

\section{Materials and methods}

Fungi

We examined four E. kreiselii isolates: LESF053 (= CBS139320), LESF303, LESF305, and LESF309. These isolates were obtained from fungus gardens of different colonies of M. morschi, collected in the municipality of Florianópolis, State of Santa Catarina, Brazil (see Meirelles et al. 2015b). The fungi were 
stored at $-80{ }^{\circ} \mathrm{C}$ as conidial suspensions in glycerol $10 \%$ and in sterile water kept at $8{ }^{\circ} \mathrm{C}$. For culture revival, conidia were surface-spread on potato dextrose agar medium (PDA, Acumedia) and incubated in the dark at $25{ }^{\circ} \mathrm{C}$ for 7 days. Three fungal cultivars were used in the bioassays: two Leucocoprinus sp. (isolates AR01 and AR02, cultivated by M. morschi), and L. gongylophorus (isolate FF2006, cultivated by the leafcutter ant Atta sexdens). Fresh cultures of the fungal cultivars were stored at $25^{\circ} \mathrm{C}$, with periodic transfer every 21 days onto the following medium (in $\mathrm{g}^{-1}$ ): 10 glucose, 5 sodium chloride, 5 peptone, 10 malt extract, 15 agar supplemented with oatmeal extract (Pagnocca et al. 1990). All fungi were periodically examined to ensure purity and viability for bioassays performance.

Dual culture assays

To evaluate the antagonistic potential of E. kreiselii isolates, we performed dual culture assays. We followed the method by Silva et al. (2006), briefly: the fungal cultivars were previously cultivated on PDA and incubated in the dark for 21 days at $25^{\circ} \mathrm{C}$. Then, fragments of $6 \mathrm{~mm}$ in diameter containing culture medium and mycelium were removed and transferred to new PDA plates $1.5 \mathrm{~cm}$ from the edge. The plates were incubated for 14 days at $25{ }^{\circ} \mathrm{C}$ before transferring E. kreiselii. Due to their slow growth rates, this period of time gave fungal cultivars a headstart to grow. Escovopsis kreiselii isolates were previously grown in the dark on PDA for 7 days. After incubation, mycelium fragments of $6 \mathrm{~mm}$ diameter from the youngest part of the colonies were removed and transferred $3 \mathrm{~cm}$ away from the host mycelium. Then, plates were incubated in the dark for 10 days at $25^{\circ} \mathrm{C}$.

Experimental controls consisted of PDA plates with hosts or E. kreiselii growing in isolation. All the interactions between the three hosts and the four Escovopsis isolates ( $\mathrm{n}=12$ combinations), as well as the respective controls $(n=7)$ were performed in ten plates each. The radial growth of colonies was recorded by scanning plates (HP Deskjet F2050, 600 dpi resolution) on days $0,1,3,5,7$ and 10 after $E$. kreiselii was transferred to plates. The mycelium growth area (in $\mathrm{cm}^{2}$ ) of all fungi was measured in ImageJ v 1.6.0_24 (Abràmoff et al. 2004).
We evaluated the effects of the dual culture interactions on both the fungal cultivars and the $E$. kreiselii isolates using three statistical tests. First, we used the non-parametric Kruskal-Wallis test to compare the mycelial growth areas of each fungal cultivar (separately) in the presence of all E. kreiselii isolates $(n=4)$ and growing alone (control). We used the final mycelial growth areas of each fungal cultivar on day 10. The Student-Newman-Keuls test was used to find the differences between the growth areas of each fungal cultivar against the treatments (E. kreiselii isolates and control).

Second, we used Mann-Whitney tests to compare the growth areas of each fungal cultivar in the presence of E. kreiselii (dual culture) and in the absence (control). As this test accounted for paired comparisons, we performed them separately for each combination (1 fungal cultivar $\times 1$ E. kreiselii isolate). In addition, within the same combination (for example: Native host 1 against E. kreiselii LESF053), we performed five tests for each day separately $(1,3,5,7$ and 10). The data set used was the mycelial growth areas of the fungal cultivar in the dual culture and alone (control). The goal of performing these tests for each combination of dual culture was to separately evaluate if there were significant differences in the growth of fungal cultivar in the presence and absence of E. kreiselii each day and to determine when inhibition occurred. In addition, we performed the same test to compare the total growth (final-initial) of each fungal cultivar in the presence and absence of each E. kreiselii isolate. Similarly, we also analysed the total growth of each $E$. kreiselii isolate against each fungal cultivar and the E. kreiselii growing alone. Finally, we used the Mann-Whitney test to evaluate when the growth stimulus started for E. kreiselii growing in the presence of each of the native hosts in comparison to E. kreiselii growing alone (control).

Thirdly, to evaluate the influence of the fungal cultivars on the mycelial growth of E. kreiselii over time, we used nparLD, a non-parametric mixed model statistical method with repeated measures (Noguchi et al. 2012). The non-parametric longitudinal data analyses are designed to study time-dependent outcomes of bioassays performed on a small number of subjects (Brunner et al. 2002), which is the case of our data set. We performed one test per E. kreiselii isolate and the data set used was the growth areas of the days $(1,3,5,7$ and 10) against each fungal cultivar $(\mathrm{n}=3)$ 
and growing alone (control), with a total of four groups (subjects) compared in each test. Thus, there were two variables involved in the tests: time (in days) and treatments (E. kreiselii in presence of each fungal cultivar or growing alone). Therefore, the model used was "F1-LD-F1", since it compares one "betweensubjects" factor (treatments) and one "within-subjects" factor (time). With this model, we calculated a Wald-type and an ANOVA-type statistics, followed by paired comparisons between treatments to evaluate the group effect. All the analyses were performed in $\mathrm{R}$ v3.5.1 (R Core Team 2018).

Fungal choice bioassays

We verified whether $E$. kreiselii directly recognized and grew towards its native hosts. Bioassays followed methods described in Gerardo et al. (2006) with modifications: plates $(150 \mathrm{~mm} \times 40 \mathrm{~mm})$ were prepared with $80 \mathrm{~mL}$ of PDA and the culture medium was cut in a cross shape. One fragment with $6 \mathrm{~mm}$ diameter containing culture medium and mycelium from each ant cultivar was separately added to the end of each track of the cross. No fungi were added to one end of the cross (control). After transferring the three fungal cultivars, plates were incubated in darkness at $25{ }^{\circ} \mathrm{C}$ for 7 days. Then, we added a fragment of $6 \mathrm{~mm}$ diameter containing culture medium and E. kreiselii mycelium to the center of the tracks. For each isolate $(n=4)$, we used nine plates, and all were incubated up to 45 days in the same conditions. The mycelial growth of all fungi was registered as described above.

To evaluate the mycelial growth of E. kreiselii towards each treatment (three tracks with fungal cultivars and one control track), we measured the distance (in $\mathrm{cm}$ ) between the mycelium and the end of each track on alternate days until the mycelium reached the end of all tracks. The measurements were made in ImageJ v 1.6.0_24 (Abràmoff et al. 2004). Then, for each E. kreiselii isolate we performed nonparametric Friedman tests with the distance measurements on day 5 and day 8 . We chose day 5 because this was the last day when growth was similar in all tracks. Day 8 was selected since it was the last day before the E. kreiselii mycelium reached the end of the tracks with the two native hosts (after day 8 the compared distances were the same). On the selected days, we compared the distance measurements (in $\mathrm{cm}$ ) between the four treatments (tracks with the three fungal cultivars and the control track). The analyses were performed in R v3.5.1 ( $\mathrm{R}$ Core Team 2018). Additionally, we calculated the growth rate of E. kreiselii in each track by dividing the distance measures on day 5 by the number of days it took to reach the end of the track after day 5. We also used the Friedman test to compare the growth rates of the four tracks for each $E$. kreiselii isolate.

Scanning electron microscopy (SEM)

We evaluated the physical interaction of E. kreiselii LESF053 with its two native hosts (Leucocoprinus sp. AR01 and AR02). We prepared three samples for each combination and the first step was to transfer a mycelium fragment of each cultivar on PDA plates and incubate it for 10 days. After incubation, a mycelium fragment of LESF053 was transferred $1.5 \mathrm{~cm}$ away from the cultivar mycelium. The dual culture was incubated at $25{ }^{\circ} \mathrm{C}$ and monitored every $12 \mathrm{~h}$ to determine the time that the hyphae contacted each other. After $48 \mathrm{~h}$, plates were fixed with a vapor of osmium tetroxide following the method by Varanda-Haifig et al. (2017). After 4 days in the osmium tetroxide, a fragment of the culture medium containing the physical interaction was cut and transferred to an aluminum holder. Then, samples were dehydrated in acetone baths with increasing concentrations: 50, 75, 90, 95 and 100\%. After critical point dehydration (Balzers CPD030), the samples were immobilized in stubs and metallised with gold sputtering (Balzers SCD050). The samples were examined under a scanning electron microscope (Hitachi TM3000). As a control, we examined the hyphae of all fungi growing in isolation in the same conditions. We used E. kreiselii reproductive structures (conidiophores and chlamydospores) to distinguish its hyphae from the host's hyphae.

\section{Results}

Host inhibition and Escovopsis kreiselii growth

Except for E. kreiselii LESF305, all isolates inhibited the growth of the three fungal cultivars by $23.95 \%$ or more when compared to the control (Table 1). In bioassays with the native hosts (Leucocoprinus sp. AR01 and AR02), the fungal cultivar mycelium 
Table 1 Mycelial growth areas (means in $\mathrm{cm}^{2} \pm$ standard error) of fungal cultivars in the presence and absence (control) of Escovopsis kreiselii (LESF053, LESF303, LESF305 and LESF309)

\begin{tabular}{|c|c|c|c|c|c|c|}
\hline Combinations & IG & FG & TG & $\begin{array}{l}\text { Day of } \\
\text { physical } \\
\text { contact }\end{array}$ & $\%$ & $P$ \\
\hline Native host AR01 (control) & $10.11 \pm 0.23$ & $22.60 \pm 0.49$ & $12.49 \pm 0.43$ & - & - & - \\
\hline x LESF053 & $10.05 \pm 0.22$ & $19.20 \pm 0.44$ & $9.15 \pm 0.29$ & 5 & 26.75 & 0.00001 \\
\hline x LESF303 & $9.80 \pm 0.14$ & $17.51 \pm 0.17$ & $7.71 \pm 0.16$ & 5 & 38.27 & 0.00001 \\
\hline x LESF305 & $10.22 \pm 0.16$ & $17.28 \pm 0.18$ & $7.06 \pm 0.17$ & 5 & 43.47 & 0.00001 \\
\hline x LESF309 & $10.01 \pm 0.24$ & $17.74 \pm 0.46$ & $7.73 \pm 0.46$ & 5 & 38.11 & 0.00004 \\
\hline Native host AR02 (control) & $7.03 \pm 0.05$ & $17.30 \pm 0.16$ & $10.27 \pm 0.15$ & - & - & \\
\hline x LESF053 & $7.01 \pm 0.13$ & $12.77 \pm 0.45$ & $5.76 \pm 0.34$ & 5 & 43.91 & 0.00001 \\
\hline x LESF303 & $7.52 \pm 0.13$ & $14.37 \pm 0.34$ & $6.85 \pm 0.32$ & 5 & 33.30 & 0.00001 \\
\hline x LESF305 & $8.02 \pm 0.33$ & $15.70 \pm 0.58$ & $7.68 \pm 0.37$ & 5 & 25.22 & 0.000206 \\
\hline x LESF309 & $7.02 \pm 0.15$ & $14.83 \pm 0.23$ & $7.81 \pm 0.29$ & 7 & 23.95 & 0.00008 \\
\hline Non-native host FF2006 (control) & $4.29 \pm 0.08$ & $9.49 \pm 0.63$ & $5.20 \pm 0.62$ & - & - & \\
\hline x LESF053 & $4.23 \pm 0.10$ & $7.84 \pm 0.25$ & $3.61 \pm 0.30$ & 10 & 30.58 & 0.02323 \\
\hline x LESF303 & $4.49 \pm 0.10$ & $7.91 \pm 0.24$ & $3.42 \pm 0.22$ & 10 & 34.23 & 0.02323 \\
\hline x LESF305 & $4.51 \pm 0.16$ & $8.77 \pm 0.26$ & $4.26 \pm 0.16$ & 10 & 18.08 & 0.123 \\
\hline x LESF309 & $4.44 \pm 0.16$ & $7.38 \pm 0.39$ & $2.94 \pm 0.37$ & 7 & 43.46 & 0.0115 \\
\hline
\end{tabular}

$I G$ initial growth means (day 0), $F G$ final growth (day 10), $T G$ total growth (FG-IG)

Inhibition percentage $\%=[1-(\mathrm{TG} \text { dual culture/TG control })]^{*} 100$

$P$ values obtained with the non-parametric test Mann-Whitney, comparing the total growth (TG) of each fungal cultivar in dual culture with Escovopsis and growing alone (control)

Figure in bold represents no significant difference

growth was inhibited after five, seven, or ten days (Table S1, Mann-Whitney, $P<0.05$ ). This inhibition only started after physical contact was established between E. kreiselii and the fungal cultivars' hyphae (Fig. 1 and Table 1).

Escovopsis kreiselii interaction with the non-native host L. gongylophorus FF2006 also resulted in antagonism. However, in all the cases, the mycelial growth inhibition of the fungal cultivar was only observed on the last day of the experiment (day 10; Table S1). Escovopsis kreiselii LESF305 was the only isolate that did not inhibit L. gongylophorus FF2006 (Table 1, Mann-Whitney, $P=0.123$ ). Moreover, considering the four E. kreiselii isolates, they were not able to overgrow the colony of this fungal cultivar in $75 \%$ (30 out of 40) of the plates. In addition, we observed a darkening of the culture medium in the contact zone between both fungi in $60 \%$ ( 24 out of 40 ) of the plates (Fig. S1).

Although the isolates belong to the same species, we found differences in the infectivity of the four $E$. kreiselii isolates against the fungal cultivars (Tables 1, $\mathrm{S} 1$ and Fig. 2). In interactions with the native host Leucocoprinus sp. AR01, E. kreiselii LESF053 was the least infective isolate, inhibiting the growth of this fungal cultivar by $26.75 \%$ compared to the control (Mann-Whitney, $P<0.05$ ). On the other hand, the remaining isolates inhibited this fungal cultivar by $38.11 \%$ or more when compared to the control. In contrast, LESF053 was the most infective isolate inhibiting an average of $43.91 \%$ of the growth of native host Leucocoprinus sp. AR02, while the other E. kreiselii isolates caused 23.95-33.30\% inhibition when compared to the control. Finally, interaction with the non-native host FF2006 resulted in greater differences between the four E. kreiselii isolates. Isolate LESF309 caused the highest inhibition of $L$. gongylophorus FF2006 growth (43.46\%), while LESF305 was the only isolate that was unable to significantly inhibit this host (18.08\%; Student-Newman-Keuls, $P=0.24$, Table 1 and Fig. 2). 


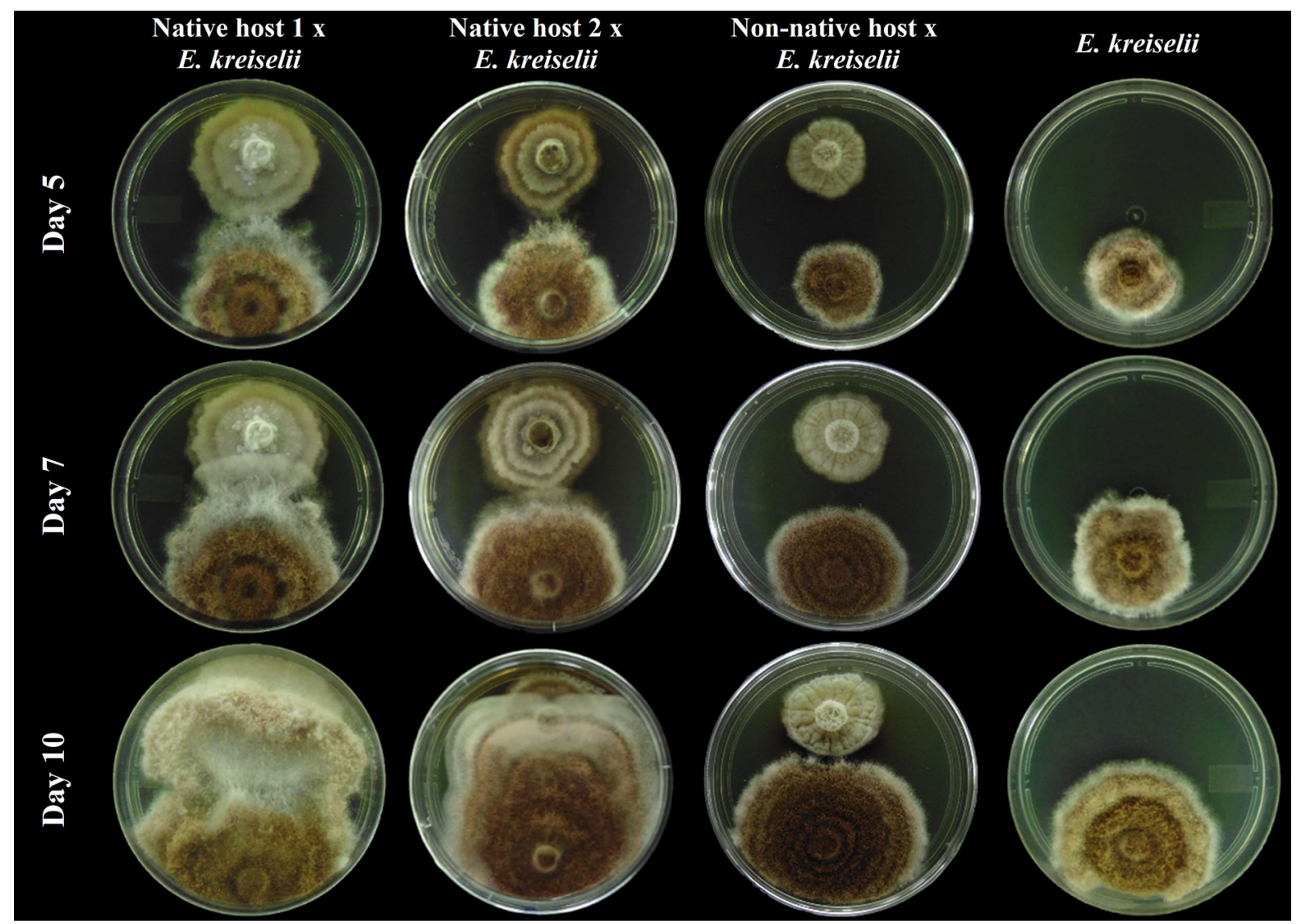

Fig. 1 Escovopsis kreiselii (LESF053) mycelial growth in the presence and absence of three fungal cultivars, in dual culture bioassays. Escovopsis kreiselii is located on the bottom of plates and the fungal cultivars on the top of plates. Native host 1 :

When E. kreiselii grew in the presence of its native hosts Leucocoprinus sp. AR01 and AR02, mycelial growth was maximised (Tables 2 and S2). All E. kreiselii isolates had a mycelial growth at least $82.96 \%$ higher in the presence of such hosts after 10 days when compared to the control (Tables 2, S2 and Fig. 3). This stimulus usually initiated before physical contact between hyphae on the first, third, or fifth day of the experiment (Mann-Whitney, $P<0.05$; Table S2 and Fig. 3). The fungal cultivar that caused the highest stimulus (101.42-122.01\%) was the native host Leucocoprinus sp. AR01, except for E. kreiselii LESF303, whose mycelial growth was more stimulated in the presence of AR02 until day seven and equally stimulated by both native hosts on the tenth day (Fig. 3). On the other hand, the non-native host $L$. gongylophorus FF2006 caused three different impacts on the growth of E. kreiselii (Table 2): (1) LESF053
Leucocoprinus sp. AR01; Native host 2: Leucocoprinus sp. AR02 and Non-native host: Leucoagaricus gongylophorus FF2006

was inhibited by this cultivar on the tenth day of dual culture (Table 2, Mann-Whitney, $P<0.05$ ); (2) LESF309 mycelial growth increased by $23.17 \%$ compared to the control (also on the tenth day; Table 2), and (3) the mycelium growth of LESF303 and LESF305 was not affected. Nevertheless, the mycelial growth of all E. kreiselii isolates in the presence of the non-native host FF2006 did not significantly differ over time from the E. kreiselii growing alone (control; paired comparisons post F1LD-F1, $P>0.05$; Table S2 and Fig. 3).

Overall, we did not observe a clear association between maximised growth and infectivity by $E$. kreiselii isolates. Figure 3 shows that the growth of $E$. kreiselii isolates LESF305 and LESF309 was higher in the presence of the same native host (AR01) that caused the highest inhibitions (Tables 1, S2 and Figs. 2, 3). On the other hand, the growth of $E$. 

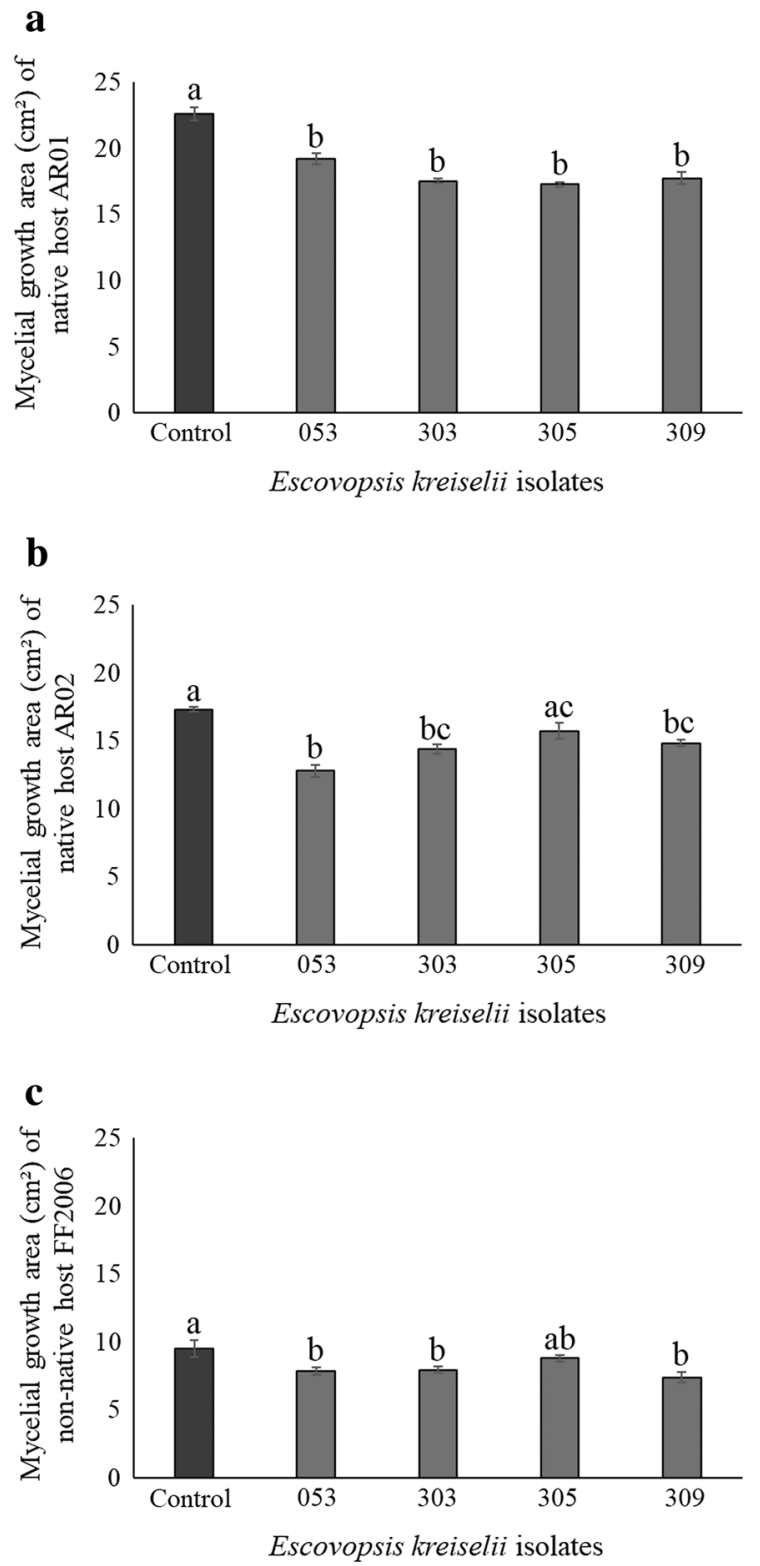

Fig. 2 Mycelial growth of three fungal cultivars (means of final areas in $\mathrm{cm}^{2} \pm$ standard errors) after 10 days in the presence and absence of E. kreiselii isolates: LESF053, LESF303, LESF305 and LESF309. Fungal cultivars: a Native host Leucocoprinus sp. AR01, b Native host Leucocoprinus sp. AR02 and c Non-native host Leucoagaricus gongylophorus FF2006. Different letters represent significant differences between treatments (Student-Newman-Keuls, $P<0.05$ )

kreiselii LESF053 in the presence of the two native hosts was similar (Table S2 and Fig. 3), although its infectivity was $17.16 \%$ higher towards native host AR02 (Table 1). In addition, E. kreiselii LESF303 showed the smallest difference between inhibitions caused in both native hosts (38.27 and 33.30\% inhibition; Table 1), but its growth was higher in the presence of AR02 from day 3 to day 7, statistically differing from its growth in the presence of AR01 $(P<0.05$, paired comparison post F1-LD-F1; Table S2 and Fig. 3).

\section{Chemical recognition of native hosts}

In fungal choice assays, all E. kreiselii isolates presented directional growth towards both native hosts (AR01 and AR02) when compared to the control (Fig. 4). After 7 days, we observed accelerated growth of isolates only towards these fungi (Table S3). Growth towards the non-native host L. gongylophorus FF2006 was the same as the control (Friedman, $P>0.05$; Table S3 and Fig. 4). There was no statistical difference between the growth rates of $E$. kreiselii towards the two native hosts. Also, we observed no differences between growth rates toward the track with the non-native host and the control (Table S3; Friedman $P>0.05$ ). Although E. kreiselii mycelium reached the cross end with $L$. gongylophorus FF2006, it did not overgrow the cultivar mycelium in the majority of plates $(72.22 \% ; 26$ out of 36$)$, (Fig. 4). This type of interaction remained even after 45 days of culture. In such cases, we also observed the darkening of the culture medium in the contact zone between fungi.

Nature of the interaction

Our SEM analysis showed the importance of the establishment of physical contact between hyphae. One out of three samples prepared per combination $(E$. kreiselii LESF053 against native host AR01 and AR02) did not provide any results, since the physical interaction was not established by the time the preparation was fixed with osmium tetroxide. Thus, we made our observations from two samples of each fungi combination. We did not observe any specialised structure for parasitism, such as hooks, wedges, fingerlike appendages, clamps or holdfasts. However, in the contact zone we observed several hyphae tangles or knots (Fig. 5a, b). In all samples, we often found $E$. kreiselii hyphae attached to the host hyphae (Fig. 5c). Such event was observed eight times in the presence of fungal cultivar AR01 and eight times in the presence 
Table 2 Mycelial growth areas (means in $\mathrm{cm}^{2} \pm$ standard error) of Escovopsis kreiselii isolates in the presence and absence (control) of ant fungal cultivars (native host AR01, native host AR02 and non-native host FF2006)

\begin{tabular}{llllr}
\hline Combinations & IG & FG & TG & $\%$ \\
\hline LESF053 (control) & $1.28 \pm 0.13$ & $26.69 \pm 0.67$ & $25.41 \pm 0.65$ & $P$ \\
x Native host AR01 & $1.74 \pm 0.13$ & $52.92 \pm 1.29$ & $51.18 \pm 1.34$ & 101.42 \\
x Native host AR02 & $1.41 \pm 0.07$ & $47.90 \pm 1.13$ & $46.49 \pm 1.09$ & 82.96 \\
x Non-native host FF2006 & $1.34 \pm 0.10$ & $23.59 \pm 0.36$ & $22.25 \pm 0.30$ & -12.44 \\
LESF303 (control) & $1.28 \pm 0.04$ & $25.08 \pm 0.77$ & $23.80 \pm 0.76$ & 0.00001 \\
x Native host AR01 & $1.23 \pm 0.07$ & $51.11 \pm 1.18$ & $49.88 \pm 1.20$ & 109.58 \\
x Native host AR02 & $1.15 \pm 0.04$ & $51.23 \pm 1.76$ & $50.08 \pm 1.75$ & 110.42 \\
x Non-native host FF2006 & $1.29 \pm 0.08$ & $25.06 \pm 1.69$ & $23.77 \pm 1.71$ & -0.13 \\
LESF305 (control) & $1.22 \pm 0.07$ & $25.47 \pm 0.49$ & $24.25 \pm 0.49$ & 0.00001 \\
x Native host AR01 & $1.92 \pm 0.10$ & $52.21 \pm 1.02$ & $50.29 \pm 0.98$ & 0.00001 \\
x Native host AR02 & $1.09 \pm 0.03$ & $49.22 \pm 1.21$ & $48.13 \pm 1.19$ & 107.38 \\
x Non-native host FF2006 & $1.29 \pm 0.08$ & $26.19 \pm 0.46$ & $24.90 \pm 0.45$ & 98.47 \\
LESF309 (control) & $1.07 \pm 0.07$ & $24.51 \pm 0.32$ & $23.44 \pm 0.28$ & 2.68 \\
x Native host AR01 & $1.03 \pm 0.05$ & $53.07 \pm 1.20$ & $52.04 \pm 1.18$ & 0.00001 \\
x Native host AR02 & $0.80 \pm 0.02$ & $46.81 \pm 3.05$ & $46.01 \pm 3.04$ & $\mathbf{0 . 4 3 5 9}$ \\
x Non-native host FF2006 & $1.74 \pm 0.17$ & $30.61 \pm 3.51$ & $28.87 \pm 3.46$ & 96.29 \\
\hline
\end{tabular}

$I G$ initial growth means, $F G$ final growth, $T G$ total growth (FG-IG)

Stimulus percentage $\%=[(\text { TG dual culture/TG control })-1]^{* 100}$

$P$ values obtained with the non-parametric test Mann-Whitney, comparing the total growth (TG) of each E. kreiselii isolate in dual culture with the fungal cultivars and growing alone (control)

Figures in bold represent no significant differences

of fungal cultivar AR02. Furthermore, we also observed strangulation of the fungal cultivars' hyphae by E. kreiselii: five times in interactions with fungal cultivar AR01 and three times in interactions with fungal cultivar AR02 (Fig. 5d). We also observed hyphal coiling in the interaction with Leucocoprinus sp. AR01 twice (Fig. 5e).

\section{Discussion}

We demonstrate the antagonistic effects of E. kreiselii on fungi cultivated by attine ants for the first time. Our results show that this fungus infects fungi cultivated by the lower attine M. morschi. The interaction between these organisms is mediated by chemical recognition. Such recognition occurs by chemotaxis: E. kreiselii presented maximum mycelial growth in the presence of its native hosts and grew towards those native hosts, even before physical contact occured. In the presence of a non-native host, E. kreiselli did not increase mycelial growth, except for isolate LESF309 that presented an increase of $23.17 \%$ when compared to the control. However, this growth stimulus was lower than the one caused by the native hosts, which boosted E. kreiselii growth by $82.96-122.01 \%$. Moreover, in cases where growth inhibition of the nonnative host fungus was observed, inhibition only occurred on the last day of the dual culture for all $E$. kreiselii isolates. Therefore, we found that infectivity and the recognition of hosts by E. kreiselii was greater against cultivars that naturally occur in the same environment from where it was isolated (in this case, colonies of M. morschi).

Inhibition of the attine ant fungal cultivars by Escovopsis spp. has been reported in several studies (Reynolds and Currie 2004; Gerardo et al. 2004, 2006; Silva et al. 2006; Folgarait et al. 2011a, b; ElizondoWallace et al. 2014; Marfetán et al. 2015; Birnbaum and Gerardo 2016; Varanda-Haifig et al. 2017). Herein, we present the first report of the antagonistic potential of E. kreiselii against fungi cultivated by the 
Fig. 3 Escovopsis kreiselii mycelial growth (means in $\mathrm{cm}^{2} \pm$ standard errors) in the presence and absence (control) of three fungal cultivars. Escovopsis isolates: a LESF053, b LESF303, c LESF305 and d LESF309. Fungal cultivars: Native host Leucocoprinus sp. AR01, Native host Leucocoprinus sp. AR02 and Non-native host Leucoagaricus gongylophorus FF2006. Different letters represent significant differences between treatments (paired comparisons post F1-LDF1, $P<0.05)$
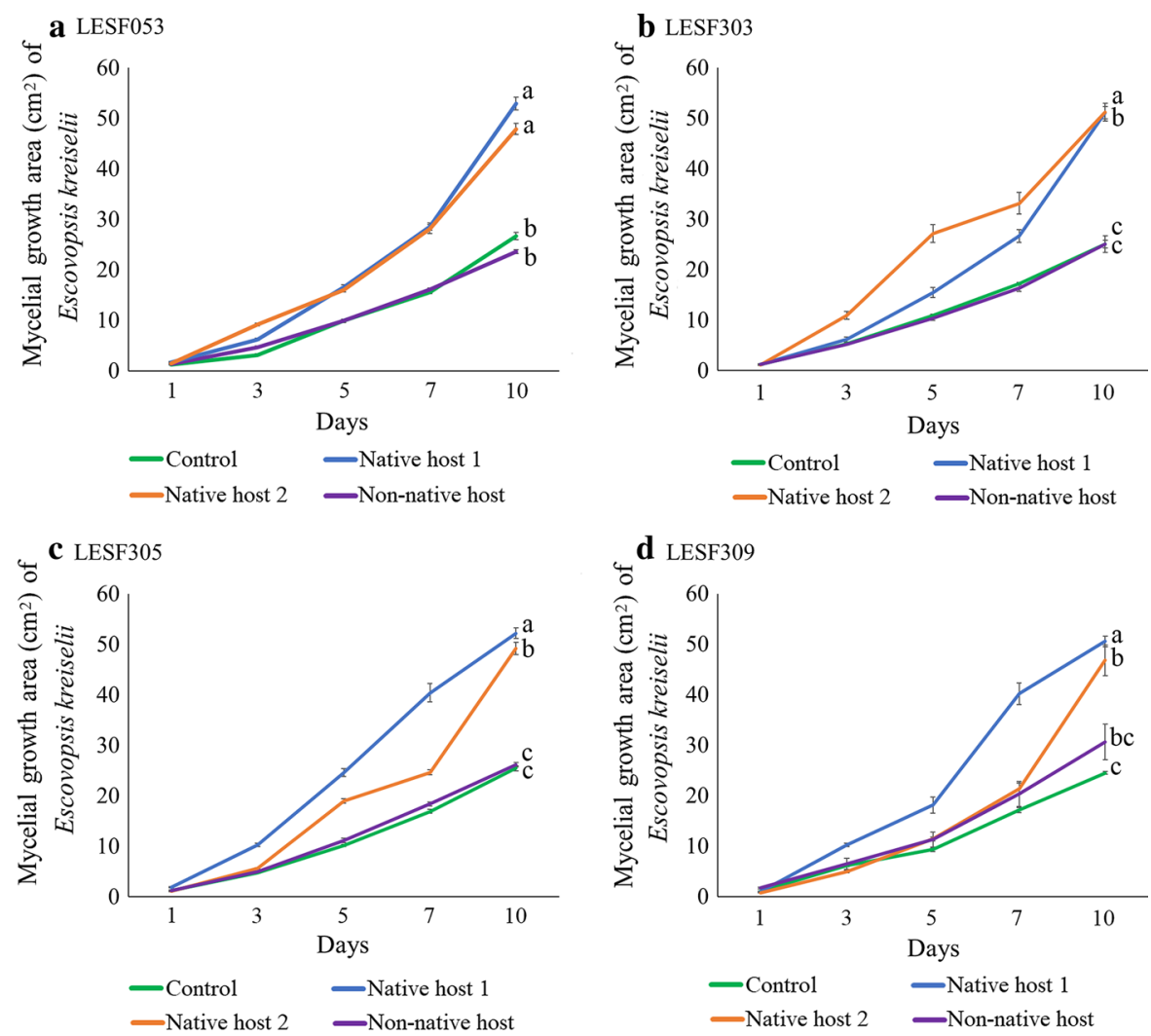

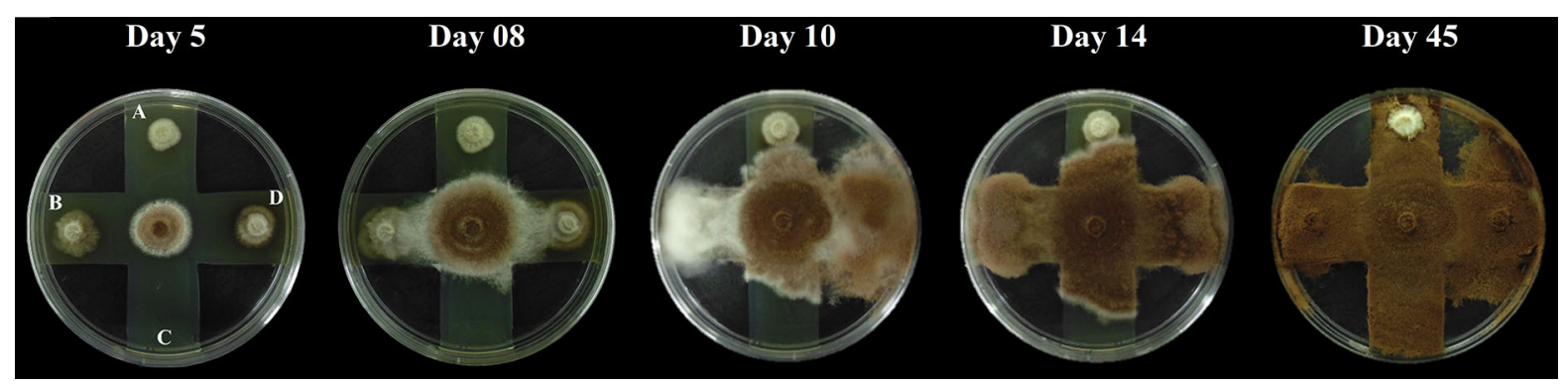

Fig. 4 Fungal choice bioassays of Escovopsis kreiselii LESF053. Fungal cultivar: a Non-native host Leucoagaricus gongylophorus FF2006, b Native host Leucocoprinus sp. AR01, c control and d Native host Leucocoprinus sp. AR02

same ant species from which it was first described $(M$. morschi). Escovopsis from colonies of higher attines affect their hosts through chemical compounds, initiating host inhibition even before physical contact between hyphae (Reynolds and Currie 2004; Folgarait et al. 2011b; Varanda-Haifig et al. 2017). A recent study identified the secondary compounds produced by Escovopsis weberi and showed that some of those compounds inhibit the in vitro development of $L$. gongylophorus (Dhodary et al. 2018). Conversely, we observed that inhibition by E. kreiselii of its native hosts was only significant after physical contact was established between both hyphae (Tables $1, \mathrm{~S} 1$ ). Thus, our results further support that physical contact is necessary for inhibition to occur. However, this does not exclude that chemical mechanisms contribute to infectivity success of E. kreiselii.

Fungi may produce metabolites for defense or attack, and some organisms use these to their advantage (Sonnenbichler et al. 1994; Folgarait et al. 2011b). Furthermore, the production of compounds and induction of sporulation may be a result of the 

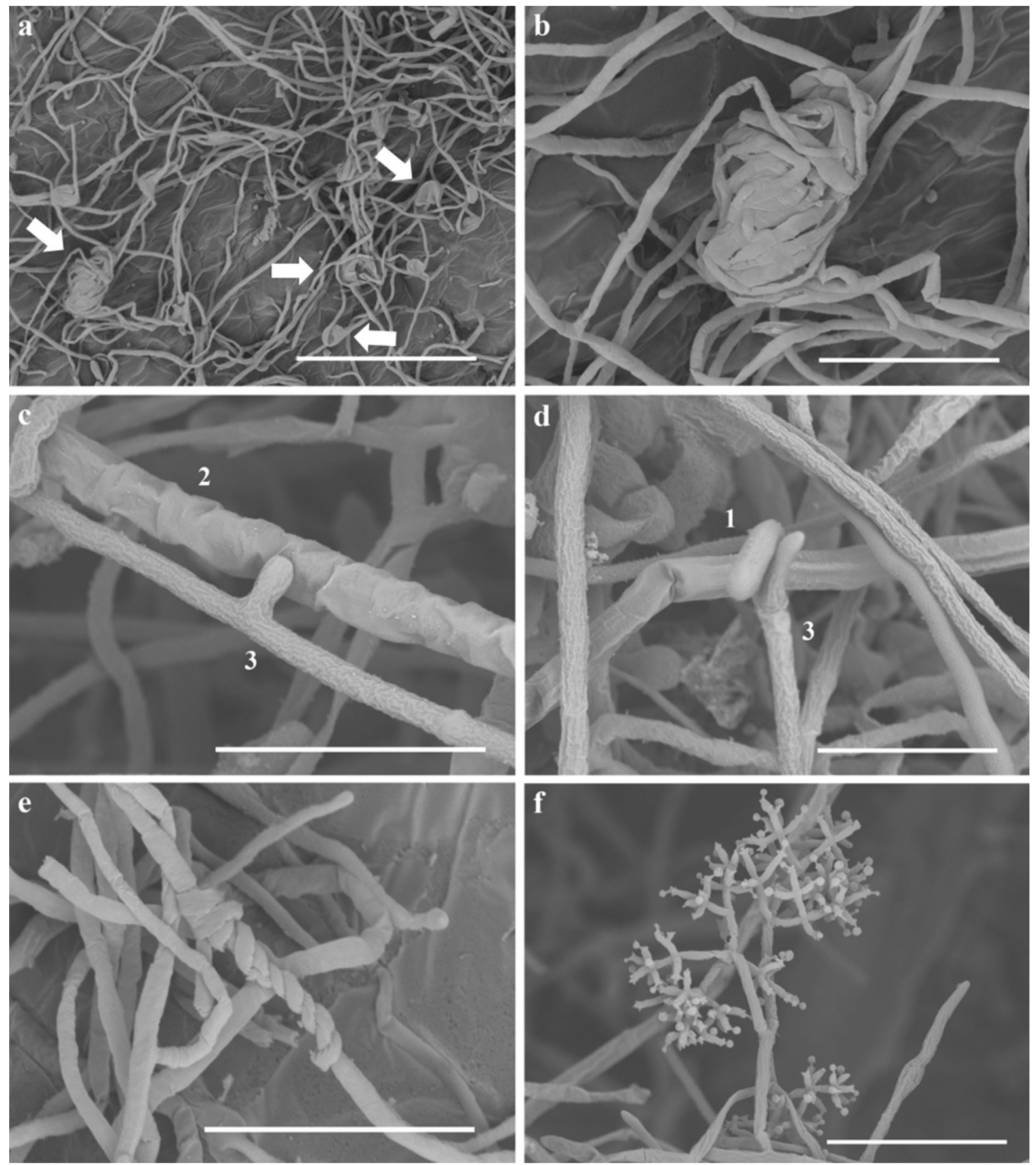

Fig. 5 Physical interaction between Escovopsis kreiselii LESF053 (indicated by \#3) and each native host: Leucocoprinus sp. AR01 (\#1) or Leucocoprinus sp. AR02 (\#2) analysed by scanning electronic microscopy. a Indicated by arrows are hyphae knots between E. kreiselii and native host AR01 (bar $100 \mu \mathrm{m})$; b Detail of a hyphae knot of E. kreiselii and native host

AR01 (bar $30 \mu \mathrm{m}$ ); c Adherence of E. kreiselii hypha to the native host AR02 hypha (bar $30 \mu \mathrm{m}$ ); d E. kreiselii hypha growing around and trapping the native host AR01 hypha (bar $30 \mu \mathrm{m})$; e E. kreiselii hypha coiling the native host AR01 hypha (bar $30 \mu \mathrm{m}$ ) and $\mathbf{f}$ Conidiophore of E. kreiselii (bar $50 \mu \mathrm{m}$ ) 
interactions between fungi (Tsujiyama and Minami 2005). In the fungal choice bioassays, we clearly observed the directional growth of E. kreiselii towards its native hosts through chemotaxis. Although we did not identify which metabolite (or metabolites) was produced by the cultivars, it was clear that soluble compounds were released by the hosts that attracted $E$. kreiselii towards them.

In the fungal choice bioassays, there was no differential preference of $E$. kreiselii between the two native hosts AR01 and AR02. However, in the dual culture bioassays we found differences in the interaction of E. kreiselii with these fungal cultivars. Several factors could account for this observation, including small variations when carrying out the experiments, as the Escovopsis mycelium fragments were more vigorous in one of the interactions, or even differences in host development. In addition, the putative evolutionary history of E. kreiselii and fungal cultivars could also account for the observed differences. In the lower attine fungiculture, cultivars are frequently exchanged between different colonies and free-living stocks, which could facilitate the horizontal transfer of Escovopsis spp. Therefore, it is possible that certain isolates had prolonged contact with one or two fungal cultivars throughout the evolution of the interaction. The E. kreiselii isolates examined and the two native hosts AR01 and AR02 were isolated from different M. morschi colonies in Florianópolis (Santa Catarina State, Brazil). Escovopsis kreiselii LESF303 and both Leucocoprinus sp. cultivars were isolated from Moçambique beach. On the other hand, the remaining E. kreiselii isolates were collected from Joaquina beach, $19 \mathrm{~km}$ away from the first site. LESF303 was the E. kreiselii isolate that showed the lowest differences in inhibition towards the two native hosts. Since these three fungi were found in the same locality (the same beach in Florianópolis), this could suggest LESF303 is in a co-evolutionary arms race with these two cultivars. On the other hand, E. kreiselii LESF305 and LESF309 were more infective and more stimulated by the same native host (AR01) and, finally, LESF053 was more infective against AR02, but was equally stimulated by both native hosts. Since these E. kreiselii isolates were obtained from a distant site as LESF303, they might have never encountered the genotypes of fungal cultivars AR01 and AR02 in nature. However, to test for potential local adaptation of certain E. kreiselii and cultivar combinations further investigation is necessary.

Our SEM experiments provided a detailed analysis of the interaction between E. kreiselii and its hosts. The main characteristic observed was the adhesion to host hyphae and the formation of hyphal knots (Fig. 5a, b, c). We believe that the formation of these structures could be a result of both attack by $E$. kreiselii and defense attempt by the host, which evidence the establishment of intimate contact between hyphae. Besides hyphal knots, we observed hyphal coiling towards the host hyphae. Mycoparasitism may involve morphological changes, and hyphal coiling could be one of them, as well as penetration and disintegration of host mycelium (AboElyousr et al. 2014). Coiling is a typical action of mycoparasites that is usually observed in some species in the genus Trichoderma (Almeida et al. 2007). Marfetán et al. (2015) observed the formation of hooks by $E$. weberi, and this structure was directly related to increased infectivity. However, this structure was not observed in E. kreiselii LESF053. Nevertheless, the infectivity of this species seems to be related to physical contact.

Host-parasite relationships may be shaped by different evolutionary processes, such as co-evolution. In the coevolutionary arms race, a host is expected to exhibit increasing resistance towards a parasite as the phylogenetic distance between the native host of such parasite increases. This mechanism is known as nonhost resistance (Antonovics et al. 2013; Birnbaum and Gerardo 2016). Our dual culture bioassay showed that in $75 \%$ of the plates with the interaction between $E$. kreiselii and the non-native host FF2006, the antagonist was not able to overgrow the cultivar mycelium. We believe that this cultivar releases soluble defense metabolites as a defense mechanism, since we observed a darkening of the culture medium in $60 \%$ of the plates, and in all of the plates E. kreiselii was not able to overgrow the fungal cultivar (Fig. S1). In the remaining $15 \%$ of the plates that E. kreiselii did not overgrow the non-native host FF2006, we did not observe the darkening of culture medium. It is possible that small amounts of this defensive metabolite (and thus not sufficient enough to cause the darkening of the culture medium) may be capable of controlling the infection by $E$. kreiselii in vitro, or perhaps other defensive metabolites that do not cause darkening of the culture medium may be involved. Thus, as 
observed by Birnbaum and Gerardo (2016) with other Escovopsis-cultivar interactions from lower attine ants, non-host resistance may be a mechanism that shapes E. kreiselii's interaction towards its host.

According to Gerardo et al. (2006), there are two adaptations in the parasite-host system that maintain Escovopsis fidelity: parasite attraction and host defense. Birnbaum and Gerardo (2016) defined patterns of host specificity, involving the attraction and inhibition of the parasite towards its host. Our results show that E. kreiselii was attracted towards the two native hosts and was not inhibited by them. Whereas in the interaction with a non-native host, E. kreiselii was not attracted to it (with one exception). Host-switching by parasites requires that parasites are able to establish contact and overcome the defenses of the new hosts (Gerardo et al. 2006). Perhaps, this is what kept $E$. kreiselii infecting $M$. morschi colonies, since it was unable to overcome the defense mechanisms (i.e. the putative release of soluble compounds) of a fungus cultivated by another ant in $75 \%$ of the plates in the dual culture assays.

\section{Conclusion}

Escovopsis kreiselii is an antagonist of the fungi cultivated by the lower attine ant M. morschi. We found recognition and attraction patterns towards its host through metabolites released by the latter, stimulating the growth of E. kreiselii before physical contact. However, these responses were weak when interacting with L. gongylophorus, the fungus cultivated by several leafcutter ant species, showing a fidelity towards cultivars that naturally occur in colonies of M. morschi. Although further studies using in vivo experiments are needed, the multiple lines of evidence presented in this study support that $E$. kreiselii is a potential parasite that likely infects fungi cultivated by $M$. morschi.

Acknowledgements We are grateful to "FAPESPFundação de Amparo à Pesquisa do Estado de São Paulo" for financial support given to AR (Grant \# 2014/24298-1) and a scholarship to BCC (\# 2015/15299-7). We also thank Fernando Carlos Pagnocca for providing isolate FF2006 of the mutualistic fungus and Irina Jiménez Gómez for technical assistance. We would like to thank Lorena Tigre Lacerda, Rodolfo Bizarria Jr, and two anonymous reviewers for providing comments on this manuscript.
Conflict of interest The authors declare that they have no conflict of interest.

\section{References}

Abo-Elyousr KAM, Abdel-Hafez SII, Abdel-Rahim IR (2014) Isolation of Trichoderma and evaluation of their antagonistic potential against Alternaria porri. J Phytopathol 162:567-574. https://doi.org/10.1111/jph.12228

Abràmoff MD, Magalhães PJ, Ram SJ (2004) Image processing with ImageJ. Biophoton Int 11:36-42

Almeida FBR, Cerqueira FM, Silva RN, Ulhoa CJ, Lima AL (2007) Mycoparasitism studies of Trichoderma harzianum strains against Rhizoctonia solani: evaluation of coiling and hydrolytic enzyme production. Biotechnol Lett 29:1189. https://doi.org/10.1007/s10529-007-9372-z

Antonovics J, Boots M, Ebert D, Koskella B, Poss M, Sadd BM (2013) The origin of specificity by means of natural selection: evolved and nonhost resistance in host-pathogen interactions. Evolution 67:1-9. https://doi.org/10.1111/j. 1558-5646.2012.01793.x

Birnbaum SSL, Gerardo NM (2016) Patterns of specificity of the pathogen Escovopsis across the fungus-growing ant symbiosis. Am Nat 188:52-65. https://doi.org/10.1086/686911

Brunner E, Domhof S, Langer F (2002) Nonparametric analysis of longitudinal data in factorial experiments. Wiley, New York

Carreiro SC, Pagnocca FC, Bueno OC, Bacci M Jr, Hebling MJA, Silva OA (1997) Yeasts associated with nests of the leaf-cutting ant Atta sexdens rubropilosa Forel, 1908. Antonie Van Leeuwenhoek 71:243-248. https://doi.org/ 10.1023/A:1000182108648

Chapela I, Rehner SA, Schultz TR, Mueller UG (1994) Evolutionary history of the symbiosis between fungus-growing ants and their fungi. Science 266:1691-1694. https://doi. org/10.1126/science.266.5191.1691

Currie CR, Mueller UG, Malloch D (1999) The agricultural pathology of ant fungus gardens. Proc Natl Acad Sci USA 96:7998-8002. https://doi.org/10.1073/pnas.96.14.7998

Dhodary B, Schilg M, Wirth R, Spiteller D (2018) Secondary metabolites from Escovopsis weberi and their role in attacking the garden fungus of leaf-cutting ants. Chem Eur J 24:4445-4452. https://doi.org/10.1002/chem.201706071

Elizondo-Wallace DE, Vargas-Ansesio JG, Pinto-Tomás AA (2014) Correlation between virulence and genetic structure of Escovopsis strains from leaf-cutting ant colonies in Costa Rica. Microbiology 160:1727-1736. https://doi.org/ 10.1099/mic.0.073593-0

Folgarait P, Gorosito N, Poulsen M, Currie CR (2011a) Preliminary in vitro insights into the use of natural fungal patoghens of leaf-cutting as biocontrol agents. Curr Microbiol 63:250-258. https://doi.org/10.1007/s00284011-9944-y

Folgarait PJ, Marfetán JA, Cafaro MJ (2011b) Growth and conidiation response of Escovopsis weberi (Ascomycota: Hypocreales) against the fungal cultivar of Acromyrmex lundii (Hymenoptera: Formicidae). Environ Entomol 40:342-349. https://doi.org/10.1603/EN10111 
Gerardo NM, Mueller UG, Price SL, Currie CR (2004) Exploiting a mutualism: parasite specialization on cultivars within the fungus-growing ant symbiosis. Proc R Soc Lond B 1550:1791-1798. https://doi.org/10.1098/rspb.2004.279

Gerardo NM, Jacobs SR, Currie CR, Mueller UG (2006) Ancient host-pathogen associations maintained by specificity of chemotaxis and antibiosis. PLoS Biol 4:e235. https://doi.org/10.1371/journal.pbio.0040235

Graczyk TK, Knight R, Tamang L (2005) Mechanical transmission of human protozoan parasites by insects. Clin Microbiol Rev 18:128-132. https://doi.org/10.1128/CMR. 18.1.128-132.2005

Horký P, Douda K, Maciak M, Závorka L, Slavík O (2014) Parasite-induced alterations of host behaviour in a riverine fish: the effects of glochidia on host dispersal. Freshw Biol 59:1452-1461. https://doi.org/10.1111/fwb.12357

Hughes DP, Andersen SB, Hywel-Jones NL, Himaman W, Billen J, Boomsma JJ (2011) Behavioral mechanisms and morphological symptoms of zombie ants dying from fungal infection. BMC Ecol 11:13. https://doi.org/10.1186/14726785-11-13

Kaiser B, Vogg G, Fürst UB, Albert M (2015) Parasitic plants of the genus Cuscuta and their interaction with susceptible and resistant host plants. Front Plant Sci 6:45. https://doi. org/10.3389/fpls.2015.00045

Kellner K, Ishak HD, Linksvayer TA, Mueller UG (2015) Bacterial community composition and diversity in an ancestral ant fungus symbiosis. FEMS Microbiol Ecol 91:fiv073. https://doi.org/10.1093/femsec/fiv073

Kellner K, Kardish MR, Seal JN, Linksvayer TA, Mueller UG (2017) Symbiont-mediated host-parasite dynamics in a fungus-gardening ant. Microb Ecol 76:530-543. https:// doi.org/10.1007/s00248-017-1124-6

Marfetán JA, Romero AI, Folgarait PJ (2015) Pathogenic interaction between Escovopsis weberi and Leucoagaricus sp.: mechanisms involved and virulence levels. Fungal Ecol 17:52-61. https://doi.org/10.1016/j.funeco.2015.04. 002

Meirelles LA, Montoya QV, Solomon SE, Rodrigues A (2015a) New light on the systematics of fungi associated with attine ant gardens and the description of Escovopsis kreiselii sp. nov. PLoS One 10:e0112067. https://doi.org/10.1371/ journal.pone.0112067

Meirelles LA, Solomon SE, Bacci M Jr, Wright AM, Mueller UG, Rodrigues A (2015b) Shared Escovopsis infections between leaf-cutting and non-leaf-cutting ants in the higher-attine fungus-growing ant symbiosis. R Soc Open Sci 2:150257. https://doi.org/10.1098/rsos.150257

Meirelles LA, McFrederick QS, Rodrigues A, Mantovani JD, de Melo Rodovalho C, Ferreira H, Bacci M, Mueller UG (2016) Bacterial microbiomes from vertically transmitted fungal inocula of the leaf-cutting ant Atta texana. Environ Microbiol Rep 8:630-640. https://doi.org/10.1111/17582229.12415

Mueller UG, Rehner SA, Schultz TR (1998) The evolution of agriculture in ants. Science 281:2034-2038. https://doi. org/10.1126/science.281.5385.2034

Mueller UG, Kardish MR, Ishak HD, Wright AM, Solomon SE, Bruschi SM, Carlson AL, Bacci M Jr. (2018) Phylogenetic patterns of ant-fungus associations indicate that farming strategies, not only a superior fungal cultivar, explain the ecological success of leafcutter ants. Mol Ecol 27:2414-2434. https://doi.org/10.1111/mec.14588

Munkacsi AB, Pan JJ, Villesen P, Mueller UG, Blackwell M, McLaughlin DJ (2004) Convergent coevolution in the domestication of coral mushrooms by fungus-growing ants. Proc R Soc Lond B 271:1777-1782. https://doi.org/ 10.1098/rspb.2004.2759

Noguchi K, Gel YR, Brunner E, Konietschke F (2012) nparLD: an $\mathrm{R}$ software package for the nonparametric analysis of longitudinal data in factorial experiments. J Stat Softw 50:1-23. https://doi.org/10.18637/jss.v050.i12

Pagnocca FC, Silva OA, Hebling-Beraldo MJ, Bueno OC, Fernandes B, Vieira PC (1990) Toxicity of sesame extracts to the symbiotic fungus of leaf-cutting ants. Bull Entomol Res 80:349-352. https://doi.org/10.1017/ S0007485300050550

Pereira JS, Costa RR, Nagamoto NS, Forti LC, Pagnocca FC, Rodrigues A (2016) Comparative analysis of fungal communities in colonies of two leaf-cutting ant species with different substratum preferences. Fungal Ecol 21:68-75. https://doi.org/10.1016/j.funeco.2016.03.004

Price PW (1980) Evolutionary biology of parasites. Princeton University Press, Princeton

R Core Team (2018) R: A language and environment for statistical computing. R Foundation for Statistical Computing, Vienna, Austria. URL https://www.R-project.org/. Accessed 25 July 2018

Reis BMS, Silva A, Alvarez MR, Oliveira TB, Rodrigues A (2015) Fungal communities in gardens of the leafcutter ant Atta cephalotes in forest and cabruca agrosystems of southern Bahia State (Brazil). Fungal Biol 119:1170-1178. https://doi.org/10.1016/j.funbio.2015.09.001

Reynolds HT, Currie CR (2004) Pathogenicity of Escovopsis weberi: the parasite of the attine-microbe symbiosis directly consumes the ant-cultivated fungus. Mycologia 96:955-959. https://doi.org/10.1080/15572536.2005. 11832895

Schultz TR, Brady SG (2008) Major evolutionary transitions in ant agriculture. Proc Natl Acad Sci USA 105:5435-5440. https://doi.org/10.1073/pnas.0711024105

Silva A, Rodrigues A, Bacci M Jr, Pagnocca FC, Bueno OC (2006) Susceptibility of the ant-cultivated fungus Leucoagaricus gongylophorus (Agaricales: Basidiomycota) towards microfungi. Mycopathologia 162:115-119. https://doi.org/10.1007/s11046-006-0037-6

Sonnenbichler JA, Dietrich J, Peipp H (1994) Secondary fungal metabolites and their biological activities, V. Investigations concerning the induction of the biosynthesis of toxic secondary metabolites in Basidiomycetes. Biol Chem 375:71-80. https://doi.org/10.1515/bchm3.1994.375.1.71

Tsujiyama SI, Minami M (2005) Production of phenol-oxidizing enzymes in the interaction between whiterot fungi. Mycoscience 46:268-271. https://doi.org/10.1007/ S10267-005-0243-Y

Varanda-Haifig SS, Albarici TR, Nunes PH, Haifig I, Vieira PC, Rodrigues A (2017) Nature of the interactions between hypocrealean fungi and the mutualistic fungus of leafcutter ants. Antonie Van Leeuwenhoek 110:593-605. https://doi.org/10.1007/s10482-016-0826-y

Weber NA (1972) Gardening ants: the Attines. American Philosophical Society, Philadelphia 